\title{
PROJETOS ORIENTADOS PARA O MERCADO: ANÁLISE EM UM CLUSTER DE TECNOLOGIA DA INFORMAÇÃO
}

\section{RESUMO}

Este artigo apresenta e analisa o resultado de pesquisa abordando o desempenho das empresas do arranjo produtivo local de Tecnologia da Informação da RMBH - MG. O modelo teórico de referência para a pesquisa-quanti/qualitativa foi o da orientação para o mercado, ou escala Markor, proposto por Kohli, Jaworski (1993). Foram pesquisados 83 empresários, de um total de 142 do setor, assim como 04 líderes de entidades empresariais. O estudo evidencia que o apoio técnico no desenvolvimento das micro e pequenas empresas proporcionado pelo SEBRAE gerou melhor nível de satisfação no construto "geração de inteligência" bem como na "resposta ao mercado", em relação ao "construto "disseminação de inteligência". O construto "resposta ao mercado das empresas atendidas" apresentou desempenho mais elevado do que o construto "disseminação das informações tecnológicas". O projeto, como um todo, foi bem avaliado pelos empresários. Nomologicamente o modelo teórico explica satisfatoriamente o desempenho do projeto.

Palavras-chave: Tecnologia da Informação; Micro e Pequenas Empresas; Orientação ao Mercado.

\section{MARKET-ORIENTED PROJECTS: ANALYSIS IN AN INFORMATION TECHNOLOGY CLUSTER}

\begin{abstract}
This article introduces and analyzes the result of research addressing the business performance of the Local Productive Arrangement of Information Technology RMBH-MG. The theoretical model of reference for the quantitative research was the Market Orientation, or Markor scale, proposed by Kohli, Jaworski (1993). 83 entrepreneurs, out of a total of 142 of the sector, as well as 04 leading business entities were investigated. The study shows that the technical support in the development of micro and small enterprises provided by SEBRAE generated better level of satisfaction in the construct "generation of intelligence" and the "reply to", in relation to "construct" dissemination of intelligence ". The construct "response to the market of the companies met" presented higher performance than the construct "dissemination of technological information". The project as a whole was well rated by entrepreneurs. Nomologicamente the theoretical model explains satisfactorily the project performance.
\end{abstract}

Keywords: Information Technology; Micro and Small Enterprises; Market Orientation. 


\section{PROYECTOS ORIENTADOS PARA EL ANÁLISIS DEL MERCADO EN UN CLUSTER DE TECNOLOGÍAS DE LA INFORMACIÓN}

\section{RESUMEN}

Este artículo presenta y analiza los resultados de las investigaciones que abordan el desempeño de las empresas en Información arreglo productivo local Tecnología BHMA - MG. El modelo teórico de referencia para la investigación cuantitativa / cualitativa fue la orientación hacia el mercado, o la escala Markor propuesta por Kohli, Jaworski (1993). 83 empresarios, un total de 142 de la industria fueron encuestados, y 04 líderes de las entidades empresariales. El estudio muestra que el apoyo técnico en el desarrollo de las micro y pequeñas empresas proporcionados por SEBRAE produce un mejor nivel de constructo satisfacción "la generación de inteligencia" y la "respuesta del mercado", en relación a "construir" la difusión de la inteligencia ". La construcción de "respuesta a las empresas del mercado servido" mostró un mayor rendimiento que el constructo de "difusión de la información tecnológica." El proyecto en su conjunto, fue bien calificado por los empresarios. Nomológicamente el modelo teórico explica satisfactoriamente el desempeño del proyecto.

Palabras clave: Tecnologías de la Información; Micro y Pequeña Empresa; Orientación al Mercado.

José Edson Lara ${ }^{1}$ Ronaldo Lamounier Locatelli ${ }^{2}$ Marcia Valéria Cota Machado ${ }^{3}$ Caissa Veloso e Sousa ${ }^{4}$

\footnotetext{
${ }^{1}$ Doutor em Administração pela Universitat Autònoma de Barcelona, Espanha. Professor da Fundação Pedro Leopoldo - FPL. Brasil. E-mail: jedson.lara@ hotmail.com

${ }^{2}$ Doutor em Economia pela University of London, Inglaterra. Professor da Fundação Pedro Leopoldo - FPL. Brasil. Email: ronaldo.lamounier@yahoo.com.br

${ }^{3}$ Mestre em Administração pela Fundação Pedro Leopoldo - FPL. Professora da Fundação Pedro Leopoldo - FPL. Brasil. E-mail: marcia.machado@sebraemg.com.br

${ }^{4}$ Doutora em Administração pela Universidade Federal de Minas Gerais - UFMG. Professora da Faculdade Novos Horizontes - FNH. Brasil. E-mail: caissa@xx.com.br
} 


\section{INTRODUÇÃO: CONTEXTUALIZANDO O ESTUDO}

Conforme tem sido abordado na literatura acadêmica, assim como na de negócios, constata-se que as organizações inseridas em mercados mais competitivos, gradualmente passam a atuar em ambientes mais sofisticados, incluindo a atuação em redes e a formação de alianças estratégicas mais sólidas. A concorrência setorial coloca-se no âmago do sucesso ou do fracasso das empresas, determinando a adequação de sua atuação, podendo, assim, contribuir para seu desempenho, sua orientação para este mercado e seu potencial inovador (Porter, 1993). Neste contexto, considera-se necessário compreender o ambiente atual que harmoniza e orquestra entidades de classe, governo, empresas, sociedade, academia e as diversas tecnologias que surgem para encurtar caminhos para o desenvolvimento. Buscar subsídios para a elaboração de uma visão compartilhada com foco em resultados nos projetos de desenvolvimento em sistemas complexos exige mais do que ouvir os principais atores que fazem parte desse contexto. É necessária a contextualização das intenções e ações em um modelo conceitual, taxonômico e nomológico que norteie e sustente estudos mais avançados no setor.

Segundo dados do Ranking Mineiro de Informática, realizado pela ASSESPRO (Associação das Empresas de Tecnologia da Informação, 2009), constata-se que o grau de envolvimento das lideranças deste APL (Arranjo Produtivo Local), tanto públicos quanto privados, está cada vez maior, trabalhando num esforço conjunto para o fortalecimento do APL e dessas empresas, perante o mercado nacional e internacional. O mesmo estudo realizado em 2011, mostra que entre os anos 2010 e 2011, o esse setor registrou crescimento de cerca de $5 \%$, superando a construção civil, e a indústria. $\mathrm{O}$ setor gerou vagas de trabalho e divisas nas telecomunicações, internet, audiovisual, edição e TI. De acordo com Lages, Braga e Morelli (2004) e Lastres e Cassiolato (2003), um Arranjo Produtivo Local - APL compreende um recorte do espaço geográfico, que possua sinais de identidade coletiva (sinais sociais, culturais, econômicos, políticos, ambientais, históricos, etc.); mantenha ou tenha capacidade de promover uma convergência em termos de expectativas de desenvolvimento; estabeleça parcerias e compromissos para manter e especializar os investimentos de cada um dos atores no próprio território; e promova, ou seja, passível de uma integração econômica e social no âmbito local.

Utilizando-se do conceito do APL, o SEBRAE MG, a partir de 2003, passou a investir nos segmentos que possuíam um maior número de empresas instaladas num mesmo território, neste caso o segmento da Tecnologia da Informação da Região Metropolitana de Belo Horizonte. De acordo com o Diagnóstico da Indústria de Software de Belo Horizonte, realizado pela Fundação João Pinheiro e pelo Serviço de Apoio às
Micro e Pequenas Empresas de Minas Gerais SEBRAE-MG, o APL de Tecnologia da Informação (TI) de Belo Horizonte, abriga cerca de 1.300 empresas que desenvolvem software e prestam serviços de informática, sendo $61 \%$ dos serviços relacionados à internet, destacando-se os de desenvolvimento de sites e de software específicos. O APL gera 17.000 empregos indiretos e 7.240 diretos, e teve um faturamento estimado de $\mathrm{R} \$ 2,8$ bilhões em 2010. São 1.300 empresas que desenvolvem software e prestam serviços de informática, sendo $61 \%$ dos serviços relacionados à internet, destacando-se os de desenvolvimento de sites e de software específicos.

Vários são os estudos que discutem os resultados do papel da tecnologia no desenvolvimento econômico das cidades. Uma das estratégias para o fomento dos projetos de desenvolvimento é o trabalho articulado em redes por meio da aglomeração de empresas, atuando no aproveitamento das sinergias geradas por suas interações, fortalecendo as chances de sobrevivência e crescimento, e constituindo-se em importante fonte de vantagens competitivas e duradouras para elas. A dinâmica instalada nos arranjos produtivos locais tem auxiliado as grandes empresas, os governos e as instituições de fomento na criação de projetos de parceria, com o intuito de estimular as empresas de micro, pequeno e médio portes a ultrapassarem as barreiras ao crescimento, a produzirem eficientemente e a comercializarem seus produtos em mercados nacionais e até internacionais.

A importância de se realizar um trabalho de análise dos resultados dos projetos desenvolvidos e da evolução dinâmica dos arranjos produtivos locais de empresas de software justifica-se não apenas pelas altas taxas de retorno que a atividade proporciona, mas principalmente pela importância das características singulares dos modelos de negócios e transversalidade deste segmento de negócios e atividades. A indústria de software assume um papel propulsor de outros segmentos de negócios, sendo importante indutora do progresso técnico. Apoiar projetos de software em contextos locais significa apoiar também outras atividades, e por isso é importante para o sucesso de ações de desenvolvimento integrado e cooperado.

Neste contexto, o objetivo geral deste estudo foi analisar o desempenho das empresas de Tecnologia da Informação (TI) da Região Metropolitana de Belo Horizonte, com o suporte proporcionado pelo SEBRAE - MG, mediante a aplicação da teoria da Orientação para o Mercado - ESCALA MARKOR.

Os objetivos específicos consistiram de:

1) Identificar e analisar as variáveis componentes dos construtos da escala MARKOR (geração de inteligência e inovação, disseminação da inteligência e resposta ao mercado);

2) Identificar e analisar o grau de associação e/ou correlação entre as variáveis dos construtos; e, 
Projetos Orientados para o Mercado: Análise em um Cluster de Tecnologia da Informação

3) Identificar e analisar a capacidade de contribuição dos construtos para a orientação.

\section{REFERENCIAL TEÓRICO: FUNDAMENTOS E MODELO TEÓRICO DO ESTUDO}

Constitui premissa deste estudo, o fato de que cada vez é mais importante compreender as tendências locacionais e distributivas do novo ciclo de expansão da economia brasileira, sua contribuição para a concentração espacial das firmas e seu impacto na redistribuição da renda e da riqueza. Haddad (2001) evidencia este crescimento econômico do Brasil por meio do surgimento de mais empregos, mais renda, mais bens e serviços. Quanto mais rápido o ritmo de crescimento, maiores as chances de se incluírem mais famílias brasileiras nos padrões civilizados de consumo privado e público. Entretanto, o crescimento econômico é uma condição necessária, mas não suficiente para o desenvolvimento deste país, já que se requer um propósito e um processo de inclusão social, com vasta gama de oportunidades e opções para as pessoas. O conceito de desenvolvimento tem sido relacionado, ao fenômeno da dinamização do crescimento econômico, mas entende-se que o crescimento econômico é necessário, mas não é suficiente para gerar desenvolvimento. Este novo ciclo apresenta-se intensivo em ciência e tecnologia na geração de diferentes produtos, processos e técnicas de gestão, que deverão compor a formação do Produto Nacional de uma economia cada vez mais exposta à competição externa.

A promoção das firmas em configuração de arranjos produtivos locais, ou clusters, ultrapassa as políticas setoriais inserindo-se no tema mais amplo do desenvolvimento regional, relacionado às novas formas de organização produtiva (LINS, 2000). Portanto, em concordância com os autores acima, pensar em desenvolvimento social, humano e sustentável, significa pensar um conceito de desenvolvimento que articula a dinamização do crescimento econômico com o crescimento do capital humano, do capital social, de uma governança estruturada e representativa e do uso sustentável do capital social. De acordo com Buarque (2002), a maioria das pessoas ainda acredita que a responsabilidade em fomentar as políticas de desenvolvimento é exclusiva do estado. Entretanto, analistas acadêmicos e autoridades das diversas áreas políticas, econômicas e sociais sustentam que haver um esforço conjunto no processo de organização social e política de uma região, mobilizar energias e recursos até então não mobilizados, nas economias locais e regionais visando a coordenação das ações de desenvolvimento.

\subsection{Arranjos Produtivos Locais}

Os aglomerados, ou arranjos de empresas, são agrupamentos geograficamente concentrados e interrelacionados, bem como instituições correlatas, com delimitação de área e vinculadas por elementos comuns e complementares, conforme afirma Porter (2002). A noção de território é importante para a atuação em Arranjos Produtivos Locais, já que a aglomeração se dá em um determinado espaço. Conceitualmente, Lages, Braga e Morelli (2004) entendem território também como um espaço definido e delimitado por e a partir de relações jurídicas, políticas ou econômicas, instituídas sempre por conformações explícitas ou implícitas de poder. Assim, o território está sempre ligado à ideia de domínio coletivo. Em Arranjos Produtivos Locais identificam-se também diferentes tipos de cooperação, incluindo a cooperação produtiva, visando à obtenção de economias de escala e de escopo, bem como a melhoria dos índices de qualidade e produtividade; e a cooperação inovadora, que resulta na diminuição de riscos, custos, tempo e, principalmente, no aprendizado interativo, dinamizando, assim, o potencial inovador do arranjo (CASSIOLATO E LASTRES, 2002). A cooperação no APL ocorre em diferentes momentos e entre diferentes atores, dentro de um processo interativo e dinâmico. Cooperação e competição coexistem no interior do arranjo produtivo.

Os Arranjos Produtivos Locais vem sendo, desde a década de 1990 definidos, contextualizados e analisados sob diversos prismas, conforme autores, como Lages, Braga e Morelli, (2004), Garofoli (1993), Campos (2004), Langlois e Robertson (1992), Villaschi Filho e Campos (2002); Santos; Diniz; Barbosa, (2004) e Arend (2012). Para estes autores, do ponto de vista do desenvolvimento social e humano sustentável, é fundamental criar um ambiente favorável ao desenvolvimento e começar investindo no capital social (quer dizer, na capacidade da sociedade de cooperar, formar redes, regular seus conflitos democraticamente e, enfim, constituir comunidade) e no capital humano (sobretudo no empreendedorismo). Sem a base de confiança fornecida pela cooperação ampliada, acumulada e reproduzida socialmente e sem empreendedorismo, dificilmente conseguir-se á, no Brasil, alavancar o desenvolvimento, como mostram numerosas evidências registradas em todas as partes do mundo. Segundo Arend (2012) as diversas trajetórias nacionais e regionais de desenvolvimento econômico dificilmente são compatíveis e comparáveis, pois cada país, região ou localidade interage uma série de fatores de natureza não só econômica, mas também social, política e cultural, que se moldam de forma específica e única. Estes diversos fatores interferem na constituição de uma rede institucional, estabelecendo formas específicas e locais do padrão de desenvolvimento. Para Bispo e Schlemm (2012), alianças estratégicas de pequenas empresas inseridas em clusters podem fortalecer a construção de suas bases de recursos, 
Projetos Orientados para o Mercado: Análise em um Cluster de Tecnologia da Informação

proporcionando-lhes melhores condições competitivas frente às grandes empresas, além de estimular a atividade empreendedora local. Entretanto, em um estudo no setor calçadista, Orssato (2012) identificou que poucas empresas agem de modo prospectivo. No geral, ainda prevalece o comportamento analista, do tipo responsivo, não pró-ativo, tendendo para a atuação em direção de nichos, com uma fraca organização de estruturas comuns, que leve à transformação da cultura corporativa em direção à cooperação. Para Alves (2012), estudando empresas comerciais em um grande shopping popular, foi verificado que a rede tem um processo de governança satisfatório, mas o compartilhamento de informações não é suficiente para gerar o aprendizado e a cooperação efetiva entre as organizações.

\subsection{Inovação e orientação para o mercado nas empresas de Tecnologia da Informação (TI)}

Outra premissa deste trabalho é que o conceito de orientação para o mercado, conforme estudado por Day (2001), Webster, 1988), Souder e Song (1997) e Kohli e Javorski (1993), pode ser estudado e aplicado na análise de organizações criadas para produzir produtos a partir de ideias inovadoras, como no caso das TI. As empresas de TI geralmente são formadas por profissionais com formação técnica ou científica (engenheiros, cientistas da computação, analistas de sistemas), que geralmente não possuem conhecimentos em gestão e marketing. Normalmente o desenvolvimento de software é uma atividade intensiva em qualificação, embora a intensidade da qualificação requerida varie entre as diferentes atividades envolvidas na produção do software. Os primeiros estágios da sua produção demandam mão-de-obra altamente qualificada, sendo a exigência de qualificação relativamente baixa nos estágios posteriores. Vale lembrar que as tecnologias de software estão presentes em virtualmente todas as atividades econômicas, mesmo em pequenas e médias empresas, especialmente nos sistemas integrados de gestão empresarial, demandando desta forma uma qualificação com conhecimento em diversos segmentos na sua concepção.

A orientação das empresas para o mercado tem sido interesse de estudo há muito tempo por parte da academia, e as pesquisas sobre o tema compreendem a investigação da adoção da orientação para o mercado, seus limites, benefícios além dos fatores facilitadores e barreiras para adoção do conceito. As empresas somente podem criar estratégias que oferecerão valor mais elevado a seus clientes e mantê-las alinhadas com as exigências de mercado se possuírem capacidade de compreender, atrair e reter clientes, características estas importantes para uma empresa orientada para o mercado (DAY, 2001). Através de metodologias de orientação para o mercado, é possível identificar informação de valor sobre o mercado alvo, e, com base nesta informação, aplicar melhorias nas características e propriedades do novo produto desde o seu desenvolvimento (Sousa, 2012).

Neste contexto, o termo orientação para o mercado pode ser entendido como um conjunto de valores e crenças que colocam o consumidor como foco principal, na elaboração da estratégia da organização (WEBSTER, 1988). Para Souder e Song (1997), a orientação para o mercado cria uma dinâmica que conduz a uma maior eficácia e eficiência no desenvolvimento das atividades da organização conduzindo para melhores resultados empresariais. $\mathrm{O}$ atual contexto mercadológico exige às empresas que considerem a inovação e a orientação ao mercado (OM) como variáveis fundamentais para um desempenho superior (Damacena, Marra e Petrol, 2012). Para tanto, muitos gestores estão utilizando a colaboração de consumidores na co-criação de valor em busca de vantagem competitiva. O fundamental, ao implementar esta nova abordagem, não é adivinhar o futuro, é cultivar uma nova cultura, baseada na construção de uma visão inspiradora do futuro que faça sentido para os diversos públicos que se relacionam com a empresa: seus clientes, as pessoas que trabalham na organização, os fornecedores, o governo etc. (Siqueira, 2009). Kohli e Javorski (1993), definiram o conceito como a geração da informação de mercado relativa as necessidades atuais e futura dos clientes em toda a organização, a disseminação da informação de mercado em todo o subsistema a organização, e a resposta a esta informação. Desta forma, identificam três componentes: (1) geração da informação, (2) disseminação da informação e (3) resposta ao mercado. A geração de informação ao mercado refere-se aos fatores externos a empresa, em que todos devem recolher e tratar as informações a respeito das necessidades dos clientes e sobre os fatores que possam afetar diretamente os desejos dos consumidores. A informação recolhida deve ser tratada e difundida pelos diversos setores da empresa para que seja conhecida e partilhada. Após a geração e disseminação da informação, surge a resposta ao mercado que se constitui na formulação e execução das ações em sinergia ao estudo de mercado. É a transformação do conhecimento adquirido em ações concretas que se refletem nas respostas às necessidades e desejos atuais e futuros dos clientes atuais e potenciais. Para os autores uma organização orientada para o mercado é aquela que assume os propósitos, processos e resultados de acordo com o conceito essencial do marketing, ou seja, suas ações são consistentes com o conceito de marketing, conforme se demonstra na figura 1 . 


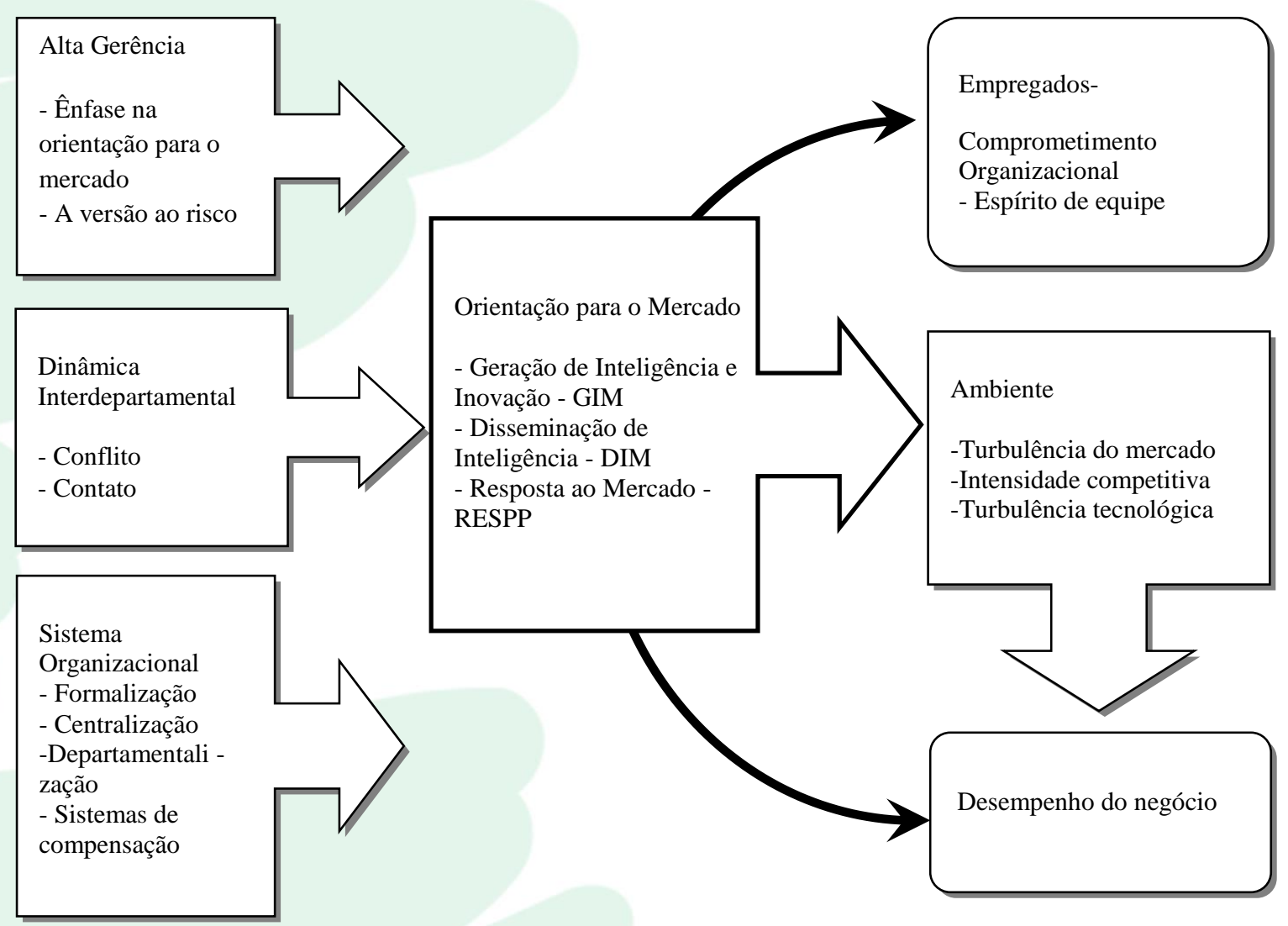

Figura 1 - Modelo de orientação para o mercado de Kohli e Jaworski Fonte: Jaworski e Kohli (1993, p.55)

Este estudo se consubstancia nos pilares do modelo acima, para estudar a orientação para o mercado, desempenhada pelas empresas de TI na RMBH, conforme explicitado nos objetivos propostos, a despeito da importância dos demais modelos de estudo. Destaca-se que, apesar de o modelo ter sido apresentado e assumido como importante contributo à compreensão da orientação para o mercado pelas empresas, não foi possível identificar algum estudo que o aplicasse ao contexto e propósitos de um APL de TI.

\section{METODOLOGIA: A TRAJETÓRIA DO ESTUDO}

Esta pesquisa se caracteriza como descritiva conclusiva, portanto, quantitativa, no propósito de se estudar as empresas mediante a aplicação estrita do modelo teórico da orientação para o mercado, ou escala de MARKOR, a partir dos seus construtos constituintes, ou seja, a geração de inteligência e inovação, a disseminação de inteligência de mercado e a resposta à inteligência de mercado para se estudar as empresas de TI. Utilizou-se a metodologia qualitativa para obter informações e percepções dos executivos das quatro instituições parceiras do SEBRAE no projeto de desenvolvimento, ou seja ASSESPRO,
FUMSOFT, SINDINFOR E SUCESU. Com este propósito, foi referenciado e adaptado o modelo experimental da pesquisa. Os construtos estabelecidos no modelo foram desmembrados em varáveis construídas especificamente para atender os propósitos desta pesquisa. Na definição das variáveis observou-se a estrita associação com seus respectivos construtos. Assim, o pressuposto é que o conjunto de variáveis explique em maior ou menor grau, cada construto. Adicionalmente o conjunto de construtos tem por propósito explicar a orientação geral do projeto ao mercado.

Como unidade de análise, foi pesquisado o projeto da Tecnologia da Informação da $\mathrm{RMBH}$ empreendido pelo Sebrae $\mathrm{MG}$ em parceria com as quatro entidades empresariais do setor. As unidades de observação foram constituídas de uma amostra 83 empresários, que se dispuseram a responder ao questionário, em um universo de 140 que foram beneficiados pelo projeto, bem como 04 líderes das entidades empresariais que representam o setor e receberam recursos financeiros para implementar as ações do projeto no APL estudado.

Para verificar o grau de orientação para o mercado dos projetos da amostra foi utilizada a escala Markor, desenvolvida por Kohli, Jaworski, conforme a Figura 1. A escala possui um total de 18 indicadores 
Projetos Orientados para o Mercado: Análise em um Cluster de Tecnologia da Informação

assim distribuídos: seis relativos à geração de inteligência de mercado (GIM), seis relativos à disseminação de inteligência de mercado (DIM) e seis relativos à resposta dos projetos (RESPP). Foi ainda elaborada uma questão com o propósito de se avaliar a orientação geral do projeto ao mercado, ou seja, às empresas atendidas. Esta pergunta assume a característica e o propósito de atuar como uma variável explicada, neste caso, pelas três variáveis explicativas: a geração de inteligência e inovação, a disseminação da inteligência e a resposta ao mercado.

Foram utilizadas, a exemplo da medida original, escalas de cinco pontos, tipo Likert, em que o entrevistado manifestou-se de acordo com o comportamento de sua empresa, variando os extremos de (1) Discordo Totalmente, a (5) Concordo Totalmente.

\section{APRESENTAÇÃO DOS RESULTADOS E ANÁLISE DOS DADOS DA PESQUISA}

\subsection{Análise quantitativa dos dados}

Conforme análise descritiva dos dados demonstram de modo geral que a atuação do SEBRAE é favorável, visto que as médias de cada grupo situaram-se próximo a 4 , em uma escala de 0 a 5 , o que demonstrando efetiva orientação da empresa para o mercado, conforme apresenta a tabela 1.

Tabela 1 - Estatísticas descritivas dos dados

\begin{tabular}{|c|c|c|c|c|}
\hline CONSTRUTO & INDICADORES & VARIÁVEIS & MÉDIA & DESVIO PADRÃO \\
\hline \multirow{6}{*}{$\begin{array}{c}\text { Geração de } \\
\text { Inteligência } \\
\text { Mercado } \\
\text { (GIM) }\end{array}$} & GIM1 & Possibilitou o desenvolvimento de inovação de produto/serviço & 4,34 & 0,825 \\
\hline & GIM2 & $\begin{array}{l}\text { Promoveu melhor nível de interação entre universidades e instituições } \\
\text { de pesquisa para geração de novas tecnologias }\end{array}$ & 3,82 & 0,934 \\
\hline & GIM3 & $\begin{array}{l}\text { Proporcionou condições para a incorporação/fusão/parceria com } \\
\text { outras empresas }\end{array}$ & 3,96 & 1,038 \\
\hline & GIM4 & $\begin{array}{l}\text { Permitiu a formação técnica e gerencial dos gestores envolvidos com } \\
\text { tecnologia e mercado }\end{array}$ & 4,11 & 0,988 \\
\hline & GIM5 & $\begin{array}{l}\text { Proporcionou a criação de tecnologias inovadoras que atendam as } \\
\text { demandas de mercado }\end{array}$ & 3,89 & 1,090 \\
\hline & GIM6 & 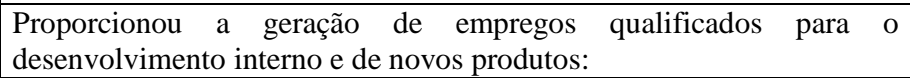 & 3,95 & 1,031 \\
\hline \multirow{6}{*}{$\begin{array}{l}\text { Dissemina- } \\
\text { ção de } \\
\text { Inteligência de } \\
\text { Mercado } \\
\text { (DIM) }\end{array}$} & DIM1 & Permitiu a melhoria da gestão dos processos internos da empresa & 3,92 & 1,197 \\
\hline & DIM2 & Permitiu a melhoria do aprendizado tecnológico da organização & 4,11 & 1,014 \\
\hline & DIM3 & Permitiu a melhoria do aprendizado estratégico e gerencial da empresa & 4,11 & 0,932 \\
\hline & DIM4 & $\begin{array}{l}\text { Permitiu a absorção de novas tecnologias de outras instituições de } \\
\text { pesquisa/empresas/universidades para o desenvolvimento do produto }\end{array}$ & 3,79 & 1,087 \\
\hline & DIM5 & Permitiu o repasse de tecnologias a outras empresas/ instituições & 3,66 & 1,014 \\
\hline & DIM6 & Permitiu o registro de patentes e marcas & 2,88 & 1,296 \\
\hline \multirow{6}{*}{$\begin{array}{l}\text { Resposta } \\
\text { Dos } \\
\text { Projetos } \\
\text { (RESPP) }\end{array}$} & RESPP1 & $\begin{array}{l}\text { Permitiu maior facilidade na captação de recursos financeiros para a } \\
\text { empresa }\end{array}$ & 3,63 & 1,164 \\
\hline & RESPP2 & $\begin{array}{l}\text { Os produtos desenvolvidos pela empresa impactaram em outros } \\
\text { segmentos de mercado }\end{array}$ & 3,89 & 0,946 \\
\hline & RESPP3 & Permitiu a melhoria da competitividade geral da empresa: & 4,18 & 0,920 \\
\hline & RESPP4 & Possibilitou a ampliação de mercados e/ou captação de novos clientes & 4,20 & 0,864 \\
\hline & RESPP5 & $\begin{array}{l}\text { Permitiu que as novas tecnologias desenvolvidas tenham tido maior } \\
\text { aderência as necessidades do mercado }\end{array}$ & 4,01 & 0,945 \\
\hline & RESPP6 & Melhorou o nível geral de satisfação dos clientes & 3,96 & 0,774 \\
\hline & OGPM & $\begin{array}{l}\text { A participação no projeto permitiu uma efetiva orientação da empresa } \\
\text { para o mercado }\end{array}$ & 4,24 & 0,728 \\
\hline
\end{tabular}

Fonte: Elaborado pelos autores.

A variável que alcançou a maior média foi a GIM1, média 4,34. Isto porque o maior investimento do SEBRAE no projeto foi para a ação de incentivo a inovação através da linha SEBRAETEC, onde foram disponibilizados mais de 1 milhão de reais durante o período. Em seguida a RESPP4, média 4,20. Entendese esta média elevada se deu em função do elevado número de rodadas de negócios e missões empresariais nacionais realizadas no período, com foco em geração de novos negócios. Quanto a variável OGPM com 
Projetos Orientados para o Mercado: Análise em um Cluster de Tecnologia da Informação

média 4,24 entende-se que o projeto na sua totalidade estava orientado para o mercado.

Quanto aos construtos, a menor média foi o construto disseminação para o mercado na variável relativa ao registro de marcas e patentes, que de alguma forma já é esperada porque este não foi o foco de atuação do projeto. Ainda nesse construto as médias referentes ao aprendizado tecnológico e gerencial foram altas, o que podem-se concluir que os esforços nas ações com este foco foram efetivos. Anualmente, o SEBRAE e seus parceiros investiram em capacitações gerenciais e técnicas para o grupo de empresas participantes, o que corrobora com o resultado da pesquisa.

Referente à variável resposta ao mercado, o construto ampliação de mercado foi também acima da média. Acredita-se que os esforços referentes à realização de rodadas de negócios e apoio à participação de feiras e missões nacionais com o objetivo de gerar oportunidades de negócios, tenham contribuído para este resultado. Dentro da mesma variável o construto referente a captação de recursos foi o menor do grupo. Entende-se este resultado em função da dificuldade das empresas em acessarem recursos para capital de giro e investimento em inovação.

Para a identificação de outliers, empregou-se o critério do número de desvios em relação à média, conforme sugerem Hair et al. (2005). Identificam-se outliers univariados pelo critério de três desvios padrão em relação à média $(|Z|>3)$. Pode-se observar que alguns indicadores apresentam alguns outliers. Este comportamento é típico das escalas utilizadas e do comportamento dos respondentes. Já os outliers multivariados foram identificados por meio da distância de Mahalanobis (D2), conforme sugerem Tabachnick e Fidel (2001). Segundo as autoras, quando existe a normalidade multivariada dos dados a distância de Mahalanobis se distribui como uma estatística quiquadrado com $k$ graus de liberdade, em que $k$ é o número de variáveis analisadas (MINGOTI, 2005). Com base nesse critério, foram encontradas 4 observações com probabilidade inferior a $0,1 \%$, sendo então a amostra reduzida para 76 casos. Os indicadores do teste de normalidade Kolmogorov-Smirnov apontam para a ausência de normalidade da maioria dos indicadores trabalhados nesta pesquisa. Este resultado é suficiente para evidenciar a violação da normalidade multivariada, já que a distribuição normal de todas as variáveis em um bloco é um requisito para a existência da normalidade multivariada de todas as combinações lineares destas variáveis (TABACHNICK e FIDEL, 2001). A verificação da unidimensionalidade dos construtos considera a homogeneidade dos itens componentes, ou seja, a quantidade de fatores ou dimensões subjacentes a uma matriz de correlações. Esse pressuposto é essencial para o cálculo de estatísticas como Alfa de Cronbach (NETEMEYER et al., 2003). A verificação da homogeneidade é normalmente feita por meio da análise fatorial exploratória (AFE) com extração de fatores por componentes principais com rotação ortogonal (varimax) (GERBING e ANDERSON, 1988; Os padrões sugeridos apontam que o número de fatores extraídos com autovalores maiores que 1 (critério de Kaiser) corresponde ao número de dimensões latentes de um conjunto de dados. O construto geração de inteligência de mercado foi formado por seis indicadores, e sua unidimensionalidade não foi confirmada pela análise fatorial, uma vez que seus indicadores apresentaram carga fatorial em duas dimensões. Além disso, alguns indicadores apresentaram carga duplicada em mais de uma dimensão, como pode ser visto na tabela 2 .

Tabela 2 - Análise da dimensionalidade do construto Geração de Inteligência de Mercado

\begin{tabular}{|c|c|c|c|}
\hline \multirow{2}{*}{ INDICADORES } & \multicolumn{2}{|c|}{ CARGA FATORIAL } & \multirow{2}{*}{ COMUNALIDADE } \\
\hline & FATOR 1 & FATOR 2 & \\
\hline GIM1 & & ,730 & 609 \\
\hline GIM2 & 572 & ,487 & ,564 \\
\hline GIM3 & & ,791 & ,629 \\
\hline GIM4 & ,537 & & ,319 \\
\hline GIM5 & ,850 & & ,741 \\
\hline GIM6 & ,807 & & 694 \\
\hline \multicolumn{3}{|c|}{ Variância Explicada } & 59,26 \\
\hline \multicolumn{3}{|c|}{$\mathrm{KMO}$} & 0,704 \\
\hline \multirow{3}{*}{\multicolumn{2}{|c|}{ Teste de Esfericidade de Bartlett }} & $\square 2$ & 82,526 \\
\hline & & $\mathrm{df}$ & 15 \\
\hline & & Sig. & 0 \\
\hline
\end{tabular}

Fonte: Elaborado pelos autores. 
Projetos Orientados para o Mercado: Análise em um Cluster de Tecnologia da Informação

Com a extração do indicador GIM2, com carga duplicada rodou-se a análise fatorial novamente, sendo mantidas as duas dimensões para o construto geração de inteligência de mercado, conforme mostra a tabela 3 .

Tabela 3 - Análise da dimensionalidade do construto Geração de Inteligência de Mercado

\begin{tabular}{|c|c|c|c|}
\hline \multirow[t]{2}{*}{ INDICADORES } & \multicolumn{2}{|c|}{ CARGA FATORIAL } & \multirow{2}{*}{ COMUNALIDADE } \\
\hline & FATOR 1 & FATOR 2 & \\
\hline GIM1 & & ,690 & ,545 \\
\hline GIM3 & & ,844 & ,713 \\
\hline GIM4 &, 575 & & 402 \\
\hline GIM5 &, 852 & & ,744 \\
\hline GIM6 & ,823 & & ,730 \\
\hline \multicolumn{3}{|c|}{ Variância Explicada } & 62,674 \\
\hline \multicolumn{3}{|c|}{ KMO } & 0,726 \\
\hline \multirow{3}{*}{\multicolumn{2}{|c|}{ Teste de Esfericidade de Bartlett }} & $\square 2$ & 52,498 \\
\hline & & df & 10 \\
\hline & & Sig. & 0,000 \\
\hline
\end{tabular}

Fonte: Elaborado pelos autores.

As duas dimensões encontradas para o construto geração de inteligência de mercado formando dois sub-construtos, o primeiro composto dos indicadores GIM1 e GIM3, e o segundo formado pelos indicadores GIM4, GIM5 E GIM6. O KMO e variância explicada se mostraram adequados.
O construto disseminação de inteligência de mercado foi formado por seis indicadores e, também, não teve sua unidimensionalidade confirmada pela análise fatorial, uma vez que seus indicadores apresentaram carga fatorial em duas dimensões, como pode ser visto na tabela 4 .

Tabela 4 - Análise de dimensionalidade do construto Disseminação de Inteligência de Mercado

\begin{tabular}{|c|c|c|c|}
\hline \multirow{2}{*}{ INDICADORES } & \multicolumn{2}{|c|}{ CARGA FATORIAL } & \multirow{2}{*}{ COMUNALIDADE } \\
\hline & FATOR 1 & FATOR 2 & \\
\hline DIM1 & ,816 & & ,676 \\
\hline DIM2 & ,845 & & ,735 \\
\hline DIM3 & ,715 & & ,527 \\
\hline DIM4 & & ,824 & ,683 \\
\hline DIM5 & 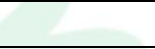 & 845 & ,726 \\
\hline DIM6 & & ,666 & ,455 \\
\hline \multicolumn{3}{|c|}{ Variância Explicada } & 73,361 \\
\hline \multicolumn{3}{|c|}{ KMO } & 0,725 \\
\hline \multirow{3}{*}{\multicolumn{2}{|c|}{ Teste de Esfericidade de Bartlett }} & $\square 2$ & 94,429 \\
\hline & & $\mathrm{df}$ & 15 \\
\hline & & Sig. & 0,000 \\
\hline
\end{tabular}

Fonte: Elaborado pelos autores.

O construto resposta dos projetos apresentou unidimensionalidade para seus seis indicadores componentes, além de KMO e variância explicada adequados aos parâmetros indicados na literatura. 
Projetos Orientados para o Mercado: Análise em um Cluster de Tecnologia da Informação

Tabela 5 - Análise da dimensionalidade do construto Resposta dos Projetos

\begin{tabular}{|c|c|c|}
\hline \multirow{2}{*}{ INDICADORES } & CARGA FATORIAL & \multirow{2}{*}{ COMUNALIDADE } \\
\cline { 2 - 3 } & FATOR 1 &, 349 \\
\hline RESPP1 &, 591 &, 365 \\
\hline RESPP2 &, 604 &, 485 \\
\hline RESPP3 &, 696 &, 718 \\
\hline RESPP4 &, 847 &, 548 \\
\hline RESPP5 &, 740 &, 403 \\
\hline RESPP6 &, 635 & 57,791 \\
\hline \multicolumn{2}{|c|}{ Variância Explicada } & 0,755 \\
\hline \multicolumn{2}{|c|}{ KMO } & 119,883 \\
\hline \multirow{2}{*}{$\begin{array}{c}\text { Teste de } \\
\text { Esfericidade de } \\
\text { Bartlett }\end{array}$} & $\square 2$ & 15 \\
\cline { 2 - 4 } & DF & 0,000 \\
\cline { 2 - 3 } & Sig. & \\
\hline
\end{tabular}

Fonte: Elaborado pelos autores.

A análise das tabelas anteriores demonstra que todos os construtos, apresentam resultados adequados ao recomendado na literatura.
A análise dos dados da tabela 6 demonstra que em todos os construtos não se recomenda a retirada de nenhum dos indicadores, que apresentaram resultados inferiores ao alfa encontrado para o construto.

Tabela 6 - Coeficiente Alfa de Cronbach para indicadores e construtos da escala

\begin{tabular}{|c|c|c|c|}
\hline CONSTRUTO & INDICADORES & $\begin{array}{c}\text { COEFICIENTE } \\
\text { ALFA }\end{array}$ & $\begin{array}{l}\text { COEFICIENTE ALFA } \\
\text { SE ITEM DELETADO }\end{array}$ \\
\hline \multirow{2}{*}{$\begin{array}{c}\text { Geração de Inteligência de Mercado } \\
1\end{array}$} & GIM1 & \multirow{2}{*}{0,710} & \\
\hline & GIM3 & & \\
\hline \multirow{3}{*}{$\begin{array}{l}\text { Geração de Inteligência de Mercado } \\
2\end{array}$} & GIM4 & \multirow{3}{*}{0,759} & ,701 \\
\hline & GIM5 & &, 528 \\
\hline & GIM6 & &, 424 \\
\hline \multirow{3}{*}{$\begin{array}{c}\text { Disseminação de Inteligência de } \\
\text { Mercado } 1\end{array}$} & DIM1 & \multirow{3}{*}{0,707} & ,623 \\
\hline & DIM2 & &, 511 \\
\hline & DIM3 & & ,699 \\
\hline \multirow{3}{*}{$\begin{array}{l}\text { Disseminação de Inteligência de } \\
\text { Mercado } 2\end{array}$} & DIM4 & \multirow{3}{*}{0,773} &, 544 \\
\hline & DIM5 & & ,482 \\
\hline & DIM6 & &, 719 \\
\hline \multirow{6}{*}{ Resposta dos Projetos } & RESPP1 & \multirow{6}{*}{0,767} & ,763 \\
\hline & RESPP2 & &, 754 \\
\hline & RESPP3 & & ,725 \\
\hline & RESPP4 & & ,686 \\
\hline & RESPP5 & &, 718 \\
\hline & RESPP6 & & ,745 \\
\hline
\end{tabular}

Fonte: Elaborado pelos autores.

Optou-se então pela permanência de todos os indicadores avaliados anteriormente pela análise fatorial e subconstrutos formados. Após a identificação da unidimensionalidade dos construtos, tornou-se 
Projetos Orientados para o Mercado: Análise em um Cluster de Tecnologia da Informação

necessário o cálculo dos mesmos. O procedimento adotado, segundo Malhotra (2004) e Hair et al. (2005) foi o cálculo dos escores fatoriais para cada caso. Os escores fatoriais consistem no produto matricial dos valores observados para as variáveis pelas cargas fatoriais. Com base nesses escores foram obtidas as médias referentes a cada construto, conforme tabela 7 .

Tabela 7 - Estatísticas descritivas para os subconstrutos

\begin{tabular}{|l|c|c|c|}
\hline \multicolumn{1}{|c|}{ SUBCONSTRUTOS } & N & MÉDIA & DESVIO PADRÃO \\
\hline Geração de Inteligência de Mercado 1 & 76 & 4,132 &, 759 \\
\hline Geração de Inteligência de Mercado 2 & 76 & 3,950 &, 815 \\
\hline Disseminação de Inteligência de Mercado 1 & 76 & 4,042 &, 844 \\
\hline Disseminação de Inteligência de Mercado 2 & 76 & 3,481 &, 879 \\
\hline Resposta dos Projetos & 76 & 4,000 &, 645 \\
\hline
\end{tabular}

Fonte: Elaborado pelos autores.

Como se pode verificar na tabela 7 , o subconstruto com maior média é o geração de inteligência de mercado 1 , com média 4,132, seguido pelo subconstruto disseminação de inteligência de mercado 1, média 4,042 e o subconstruto resposta dos projetos, média 4,000. Os subconstrutos com menores médias foram disseminação de inteligência de mercado 2, média 3,481 e geração de inteligência de mercado 2, média 3,950 .

\section{ANÁLISE DAS CORRELAÇÕES ENTRE AS VARIÁVEIS}

Para se atender um dos objetivos deste trabalho, foram calculadas as correlações entre as variáveis componentes de cada construto, como pode ser verificado nas tabelas a seguir.

Tabela 8 - Coeficientes de correlação entre as variáveis do construto Geração de Inteligência

\begin{tabular}{|l|c|c|c|c|c|}
\hline CORRELAÇÕES & GIM1 & GIM3 & GIM4 & GIM5 & GIM6 \\
\hline GIM1 & 1 & & & & \\
\hline GIM3 &, $265^{*}$ & 1 & & & \\
\hline GIM4 & 0,168 & 0,173 & 1 & & \\
\hline GIM5 & 0,129 & $-0,004$ &, $270 *$ & 1 & \\
\hline GIM6 &, $335^{* *}$ & 0,16 &, $359^{* *}$ &, $540 * *$ & 1 \\
\hline
\end{tabular}

OBS: * Correlação é significativa no nível 0.05 (bi-caudal)

**.Correlação é significativa no nível 0.01 (bi-caudal)

Fonte: Elaborado pelos autores.

Conforme verificado na tabela 8 , as correlações entre as variáveis do construto geração de inteligência de mercado não são muito elevadas. A maior se estabelece entre as variáveis GIM5 e GIM6
(0,540 significativa no nível 0,01). A variável GIM3, por outro lado, somente se correlaciona significativamente com a variável GIM1(0,265 significativamente no nível 0,05 ).

Tabela 9 - Coeficientes de correlação entre as variáveis do construto Geração de Inteligência

\begin{tabular}{|l|c|c|c|c|c|c|}
\hline CORRELAÇÕES & DIM1 & DIM2 & DIM3 & DIM4 & DIM5 & DIM6 \\
\hline DIM1 & 1 & & & & & \\
\hline DIM2 &, $545^{* *}$ & 1 & & & & \\
\hline DIM3 &, $354^{* *}$ &, $454^{* *}$ & 1 & & & \\
\hline DIM4 &,- 064 &, 032 &, 114 & 1 & & \\
\hline DIM5 &,- 012 &, 204 &, 180 &, $563^{* *}$ & 1 & \\
\hline DIM6 &, 071 &, 202 &, 010 &, $323^{* *}$ &, $385^{* *}$ & 1 \\
\hline
\end{tabular}

OBS: * Correlação é significativa no nível 0.05 (bi-caudal)

**.Correlação é significativa no nível 0.01 (bi-caudal)

Fonte: Elaborado pelos autores. 
Projetos Orientados para o Mercado: Análise em um Cluster de Tecnologia da Informação

A análise das correlações entre as variáveis componentes do construto disseminação de inteligência de mercado aponta a formação de dois grupos distintos de correlações significativas. O primeiro formado pelas variáveis DIM1, DIM2 e DIM3, onde a maior correlação é entre as variáveis DIM1 e DIM2 (0,545 significativa no nível 0,01). No segundo grupo, formado pelas variáveis DIM4, DIM5 e DIM6, a maior correlação é entre as variáveis DIM4 e DIM5 (0,563 significativa no nível 0,01 ).

Tabela 10 - Coeficientes de correlação entre as variáveis do construto Resposta dos Projetos

\begin{tabular}{|l|c|c|c|c|c|c|}
\hline & RESPP1 & RESPP2 & RESPP3 & RESPP4 & RESPP5 & RESPP6 \\
\hline RESPP1 & 1 & & & & & \\
\hline RESPP2 &, 194 & 1 & & & & \\
\hline RESPP3 &, $438^{* *}$ &, $268^{*}$ & 1 & & & \\
\hline RESPP4 &, $338^{* *}$ &, $417^{* *}$ &, $558^{* *}$ & 1 & & \\
\hline RESPP5 &, $356^{* *}$ &, $270^{*}$ &, $365^{* *}$ &, $617^{* *}$ & 1 & \\
\hline RESPP6 &, 206 &, $431^{* *}$ &, 217 &, $450^{* *}$ &, $365^{* *}$ & 1 \\
\hline
\end{tabular}

OBS: * Correlação é significativa no nível 0.05 (bi-caudal)

**.Correlação é significativa no nível 0.01 (bi-caudal)

Fonte: Elaborado pelos autores.

As correlações entre as variáveis formativas do construto resposta dos projetos não são muito elevadas. A maior correlação é identificada entre as variáveis RESPP4 e RESPP5 (0,617 significativa no nível

$0,01)$.

Tabela 11 -

Coeficiente de correlações entre os construtos

\begin{tabular}{|l|c|c|c|c|}
\hline CONSTRUTOS & GIM & DIM & RESPP & OGPM \\
\hline GIM & 1 & & & \\
\hline DIM &, $604^{* *}$ & 1 & & \\
\hline RESPP &, $672^{* *}$ &, $661^{* *}$ & 1 & \\
\hline OGPM &, $361^{* *}$ &, $358^{* *}$ &, $453^{* *}$ & 1 \\
\hline
\end{tabular}

OBS: **.Correlação é significativa no nível 0.01 (bi-caudal)

Fonte: Elaborado pelos autores.

\section{ANÁLISE DA REGRESSÃO ENTRE OS CONSTRUTOS}

A análise de regressão múltipla tem por objetivo, estimar o impacto do incremento de cada variável independente - que se traduz no peso de cada variável independente - sobre a respectiva variação da variável dependente. Os pesos denotam a contribuição relativa das variáveis independentes para a previsão geral e facilitam a interpretação sobre a influência de cada variável explicativa em fazer a previsão apontam Hair et al (2005). No caso específico desta pesquisa, procurou-se identificar e avaliar a contribuição dos construtos geração de inteligência de mercado, disseminação da inteligência de mercado e resposta ao projeto para a orientação geral do projeto ao mercado. Sendo assim, a variável dependente do modelo de regressão múltipla seria a orientação ao mercado e as variáveis independentes os demais construtos.

Como na primeira rodagem uma parte dos dados não apresentou peso estatisticamente significativo na explicação da OGPM, elas foram retiradas e uma nova regressão foi construída. No novo modelo o R2 aumentou para 0,292, mas é ainda considerado baixo para explicar a variável OGPM. Os pesos das variáveis na explicação do modelo são demonstrados na tabela 12 a seguir. 
Tabela 12 - Coeficientes da regressão múltipla do modelo corrigido

\begin{tabular}{|l|c|c|c|c|c|}
\hline \multirow{2}{*}{$\begin{array}{c}\text { VARIÁVEIS } \\
\text { PREDITORAS }\end{array}$} & \multicolumn{2}{|c|}{$\begin{array}{c}\text { COEFICIENTES NÃO } \\
\text { PADRONIZADOS }\end{array}$} & $\begin{array}{c}\text { COEFICIENTES } \\
\text { PADRONIZADOS }\end{array}$ & \multirow{2}{*}{ t } & \multirow{2}{*}{ Sig. } \\
\cline { 2 - 4 } & $\mathrm{B}$ & Erro Padrão & Beta & & \\
\hline (Constant) & 2,414 &, 379 & & 6,378 &, 000 \\
\hline DIM1 &, 178 &, 072 &, 293 & 2,483 &, 015 \\
\hline DIM2 &, 252 &, 089 &, 352 & 2,835 &, 006 \\
\hline RESPP4 &, 354 &, 096 &, 420 & 3,697 &, 000 \\
\hline
\end{tabular}

a. Variável dependente: OGPM

Fonte: Elaborado pelos autores.

Como se pode observar, a variável RSPP4 (Possibilitou a ampliação de mercados e/ou captação de novos clientes) é a que tem maior peso na explicação da orientação geral para o mercado (beta igual a 0,42 ), seguida da variável DIM2 (Permitiu a melhoria do aprendizado tecnológico da organização) com beta igual a 0,352 e da variável DIM1 (Permitiu a melhoria da gestão dos processos internos da empresa) com beta igual a 0,293 .

\section{DISCUSSÃO}

No que tange à aplicabilidade e validação do modelo teórico, o estudo evidencia que o apoio técnico no desenvolvimento das micro e pequenas empresas proporcionado pelo SEBRAE gerou melhor nível de satisfação no construto "geração de inteligência" bem como na "resposta ao mercado", em relação ao "construto "disseminação de inteligência". O construto "resposta ao mercado das empresas atendidas" apresentou desempenho mais elevado do que o construto "disseminação das informações tecnológicas". O projeto, como um todo, foi bem avaliado pelos empresários $\mathrm{O}$ modelo suportou as validações discriminantes e convergentes, necessárias à aplicabilidade neste setor. Nomologicamente o modelo teórico explica satisfatoriamente o desempenho do projeto, permitindo recomendá-lo à aplicação em estudos posteriores.

O tema da orientação para o mercado conforme analisado nesta pesquisa, permite concluir que a maior problemática no setor é como acessar novas fontes de recursos para empresas intensivas em tecnologia. O "gap" entre desenvolvimento do produto e sua inserção no mercado é grande, impactando negativamente no fluxo de caixa das organizações. Em função dos esforços dos vários stakeholders que apoiam o projeto, recentemente o BDMG criou a linha Pró-inovação, composta por juros mais baixos que os de mercado, maior prazo de carência e sem a necessidade de garantia real. Provavelmente, a partir de 2012 este construto poderá obter um melhor resultado, na variável focada em ampliação e captação de recursos.

O melhor resultado constatado pela pesquisa, na análise dos construtos foi a Inteligência para o Mercado, na variável relativa ao desenvolvimento de inovação de produtos e serviços que, inclusive, vem ao encontro de um dos objetivos da instituição que é aumentar o grau de inovação das micro e pequenas empresas e fortalecer o acesso das MPE aos mercados interno e externo, o que corrobora com o propósito de promover a competitividade e o desenvolvimento sustentável das micro e pequenas empresas e fomentar o empreendedorismo. A partir do levantamento das prioridades setoriais estabelecidas para a instituição, buscou-se a consolidação das prioridades setoriais do Estado, tendo sido utilizado como critério o grau de recorrência de tais prioridades, e do ponto de vista dos territórios, segundo o grau de relevância das iniciativas desenvolvidas pela instituição e parceiros.

Por ser inovador por natureza e demandar o desenvolvimento de novas tecnologias constantemente, o setor de Tecnologia da Informação foi selecionado, pelo grau de relevância para a consecução da estratégia de atuação do SEBRAE-MG, definindo como fatores relevantes o desenvolvimento de soluções educacionais que viabilizem o incremento do empreendedorismo; o desenvolvimento de soluções de inovação focadas no aumento da competitividade das MPEs e o desenvolvimento de soluções de acesso a mercados que promovam a internacionalização das empresas do segmento.

Pode ser visto como um grande desafio atuar nestes novos modelos, pois Belo Horizonte conta atualmente com poucas empresas que desenvolvem tecnologias focadas em saúde e mobilidade, em relação a quantidade total de empresas instaladas. Trata-se de um grupo diversificado onde coexistem empresas inovadoras que são competitivas nos mercados regionais (América Latina) e empresas cuja capacidade de inovação não foi identificada.

Embora nem todas as empresas do APL estejam tecnologicamente preparadas, estas empresas se deparam com um aumento significativo na demanda 
Projetos Orientados para o Mercado: Análise em um Cluster de Tecnologia da Informação

por suas soluções de TI para diversos setores tanto no curto quanto no médio e longo prazo.

Como pontos fortes do APL destacam-se o conhecimento da realidade local por parte das empresas, isto é, a capacidade de dar respostas adequadas às especificidades das cidades brasileiras/latino-americanas e a reconhecida qualidade da mão-de-obra formada em informática e ciência da computação pelas universidades e centros universitários da cidade e região. Além disso, outro fator relevante é a concentração de empresas de TIC em Belo Horizonte e na RMBH o que contribui para geração de um ambiente propício para a cooperação e competição. Há ainda que ressaltar a presença de centros de excelência em conhecimento como a UFMG e a PUC Minas e de uma série de instituições de apoio e fomento às indústrias e setores envolvidos em soluções para mobilidade e saúde.

Como pontos negativos do APL destacam-se por parte das empresas ausência de visão e direcionamento estratégicos, pois o estudo mostrou que no construto geração de Inteligência a maior média foi a geração de inovação para novos produtos e serviços, mas ao mesmo tempo neste mesmo construto a menor média foi o desenvolvimento de produtos inovadores de acordo com a demanda de mercado. Portanto, entende-se que as empresas tem dificuldade de interlocução com a demanda. Percebe-se no estudo a aversão a riscos por parte da maioria das empresas, especialmente na tomada de empréstimos para desenvolvimento de novos produtos, pois as mesmas ancoram-se em recursos subvencionados.

No estudo pode-se observar também uma falta de sinergia entre as universidades, as instituições de apoio e as empresas do polo, impactando de forma negativa, sobre o dinamismo tecnológico das organizações dificultando o reconhecimento do polo como referência no mercado. A expectativa é que a interação universidade-empresa possa ser exercida pelo parque tecnológico de Belo Horizonte - BHTEC. Tal relacionamento é fundamental ao desenvolvimento do APL, principalmente poderá contribuir para ser reconhecido como liderança no mercado.

Apesar do baixo relacionamento cooperativo entre as entidades, ele existe também entre as empresas. Durante o estudo observou-se que nas ações onde prevalece a cooperação as empresas se saíram melhor que o trabalho individual. A troca de experiências e o estímulo a cooperação torna as pequenas empresas mais seguras e sólidas no mercado. Algumas empresas ignoram a importância da atuação conjunta entre si, com o governo e com as universidades, esperando por um "paternalismo" por parte do poder público o que contradiz a lógica de estímulo a competitividade nas organizações.

De um modo geral, a especialização do APL com o desenvolvimento de produtos e serviços de TI com alto valor agregado, a sua localização geográfica dada por limites claros, observando o município de
Belo Horizonte com transbordamento no seu entorno, sua significativa participação na produção e exportação de TI nacional, a elevada qualidade da sua mão-deobra, apesar de escassa, e a interação das empresas com os atores locais, tanto públicos quanto privados, permitem a caracterização de um APL de empresas de TI de Belo Horizonte e Região Metropolitana, com empresas que se desenvolvem numa lógica de orientação para o mercado. Os empresários avaliam a parceria do SEBRAE no projeto, de forma geral, no que se refere ao desenvolvimento dos projetos para TI como relevante, pois o SEBRAE participou fortemente das ações com projetos de apoio que proporcionaram o envolvimento e maior conhecimento por parte dos gestores a respeito do setor. Por esta experiência, a contribuição foi mais significativa. O empresário refere-se ao APL como referência nacional, seja na geração de casos de sucesso, no incremento do potencial competitivo e principalmente no avanço das inter-relações: EMPRESA, MERCADO, GOVERNO, e aponta a nova meta, que é transformar Belo Horizonte na capital brasileira de TI até o ano de 2022.

\section{CONSIDERAÇÕES FINAIS}

Ainda que a unidade analítica deste estudo, o Polo de TI da RMBH, ser o maior do país em número de empresas, a pesquisa permitiu cobrir apenas $5 \%$ do total delas, pois o foco são as aquelas que participaram deste projeto. Assim, considera-se conveniente estudar também empresas que nunca participaram de projetos de desenvolvimento apoiados com recursos públicos, e realizar uma pesquisa comparativa, com metodologias pertinentes. Desta forma, será possível verificar o nível de prontidão das empresas de cada grupo específico. $\mathrm{O}$ estado de desenvolvimento tecnológico e gerencial, assim como as condições ambientais, político-legais e mercadológicas do setor de TI, notadamente na RMBH permitem, e convidam, a realização de estudos consistentes que objetivam a análise de suas estratégias, estruturas, bem como de outras frentes de resultados.

Algumas abordagens provindas das mídias da literatura regional de negócios tem possibilitado constatar que o setor de TI tem crescido em menor proporção que outros setores da economia, mesmo sendo portador de boas perspectivas futuras. Portanto, recomenda-se, para fomentar o crescimento das empresas instaladas no polo, que as lideranças públicas e privadas devam intensificar esforços no desenvolvimento de uma política de governo integrada na RMBH através do alinhamento do setor com os setores público, privado e academia. As iniciativas, algumas pioneiras, do Projeto BHTEC são consistentes e robustas. Entretanto, são, notoriamente, insuficientes para apoiar as empresas que se situam fora do projeto. As entrevistas revelaram alguns gaps neste quesito. Este tem sido um setor econômico que depende 
substancialmente dos esforços da chamada Tríplice Hélice, para alavancar o crescimento, em razão de ser agente muito ativo na economia do conhecimento. Alguns entrevistados revelaram também, espontaneamente, que o estado vem perdendo grandes consumidores de TI, tais como em Telecomunicações, Aço, Siderurgia e Mineração migrando a demanda para o Rio de Janeiro e São Paulo. Este fato corrobora a queda de competitividade do polo em relação aos dois estados. Talvez as criações de centros de especializações em TI direcionadas aos segmentos reconhecidos em Minas Geriam tais como a indústria automotiva e da saúde podem contribuir para elevar a competitividade de Minas Gerais, em relação aos outros estados.

Em algumas variáveis do estudo pode-se constatar que a heterogeneidade das empresas pode impactar no resultado final de algumas ações, principalmente aquelas voltadas para inovação, mercado e gestão. Foi verificada também a importância da implementação de um programa de fortalecimento da governança do polo. Estruturar um sistema de governança voltado para a coordenação e gestão das iniciativas do APL de TI da RMBH, poderá elevar o nível de atendimento dos propósitos dos stakeholders que apoiam as iniciativas do polo.

$\mathrm{O}$ estudo permitiu inferir que a diversidade de segmentos tanto de produtores de software e de serviços, como do setor usuário torna difícil estabelecer diretrizes e prioridades convergentes face aos recursos existentes, disponíveis e mobilizáveis. Entretanto esta realidade configura e determina elementos estruturais de robustez do setor, a longo prazo. Observa-se também que a ausência de um órgão coordenador do cluster, capaz de estudar e estabelecer políticas, diretrizes e prioridades dificulta o seu desenvolvimento, a exemplo do Farol Digital em Pernambuco e do Porto Digital em Recife, o que não acontece hoje com o polo da RMBH.

Quanto ao desenvolvimento substantivo do conhecimento neste setor, recomenda-se, fortemente, que novos estudos sobre o polo devam ser realizados. Neste propósito, novos modelos teóricos, novas possibilidades morfológicas e novas configurações métricas são possíveis no sentido de se estudar, com consistência, este importante campo de conhecimentos e de atividades, assim como sua orientação ao mercado. Adicionalmente, recomenda-se a replicação do mesmo modelo a outras realidades, buscando as necessárias validações discriminantes, convergentes e nomológicas, que uma estrutura de conhecimento em evolução requer. Outra fronteira de estudos consiste em estabelecer grupos de controle para observar novos resultados ou novos modelos de negócios, comparados com outros enclaves desta campo industrial.

Para a realização deste estudo encontrou-se uma dificuldade fundamental na geração da base de dados analíticos, ou seja, a resistência de diversos executivos que participaram do projeto em responder a pesquisa, reduzindo, desta forma, o universo de empresas estudas.

\section{REFERÊNCIAS}

Alves, R.C. (2012) Formação de estratégias em micro e pequenas empresas: um estudo no Mercado Central de Belo Horizonte-MG. Tese de doutorado apresentada à Universidade Federal de Lavras.

Arend, M; Cario, S. A. F.; Enderle, R. (2012) Instituições, inovações e desenvolvimento econômico. Pesquisa \& Debate. Revista do Programa de Estudos Pós-Graduados em Economia Política. SP, volume 23, número 1(41) p. 110-133

Assespro (2009). Ranking Mineiro de Informática. Disponível em www.assespro-mg.org.br Acesso em 30/10/2010.

Bispo, C. M.; Schlemm, M. M. (2012) Alianças em clusters: uma estratégia para a construção da Base de Recursos. Perspectivas Contemporâneas 7.2

Buarque, S. C (2002). Construindo o desenvolvimento local sustentável metodologia de planejamento. Rio de Janeiro: Garamond Universitária.

Campos, A (2004). Arranjos Produtivos no Estado do Paraná: o caso do município de Cianorte. Tese de Doutorado em Desenvolvimento Econômico, Universidade Federal do Paraná, Curitiba, PR, Brasil.

Cassiolato, J. E.; Lastres, H. M. M (2002). O enfoque em sistemas produtivos e inovação locais. In: FISCHER, T. (Org.). Gestão do desenvolvimento e poderes locais: marcos teórico e avaliação. Salvador: Casa da Qualidade. p. 61-76.

Damacena, C.; Marra, G. S.; Petroll, M. M. (2012) Orientação ao mercado, inovação colaborativa com os consumidores e sobrecarga de informação. Perspectivas Contemporâneas 7.2.

Day, G. S (2001). A Empresa Orientada Para o Mercado. 1a Ed. Porto Alegre: Bookman.

Didonet, S. R.; Lara, J. E.; Jimenez, D. P. (2006) Eficiência Produtiva y Estratégias en la Distribución Comercial: El Caso de los Supermercados Brasileños. ENANPAD 2006 Salvador.

Gerbing, D. W.; Anderson, J. C. (may 1988) An Updated Paradigm for Scale Development Incorporing Unidimensionality and it's Assesssment. Journal of Marketing Research, v. 25, p. 186-192. 
Projetos Orientados para o Mercado: Análise em um Cluster de Tecnologia da Informação

Haddad, P. R (2001). Clusters e Desenvolvimento Regional no Brasil. In: Revista Cluster, n. 2, Belo Horizonte: Instituto Metas.

Hair Jr. J. F. et al. (2005) Análise Multivariada de Dados. $5^{\text {a }}$. Ed. Porto Alegre: Bookman.

Jaworski, B.J. e Kohli, Ajay K. (July 1993), Market Orientation: Antecedents and Consequences, Journal of Marketing, p.53-70.

Lages V.; Braga C.; Morelli, G. (2004). Territórios em movimento: cultura e identidade como estratégia de inserção competitiva, in Frando, A.- Pobreza e desenvolvimento local. Brasília, Editora Relume Dumará.

Langlois, R. N. \& Robertson, P. L. Firms (1995), Markets and Economic Change: A Dynamic Theory of Business Institutions. Routledge, London.

Lastres, H.M.M; Cassiolato, J. E. (2003). Novas políticas na Era do Conhecimento. O foco em arranjos produtivos e inovativos locais. Revista Parcerias Estratégicas, n.17, p. 05-29, setembro.

Malhotra, N. (2004) Pesquisa de Marketing: uma orientação aplicada. 4 ${ }^{\mathrm{a}}$. Ed. Porto Alegre. Ed. Bookman.

Mingoti, S. (2005) Análise de dados através de métodos de pesquisa multivariada: uma abordagem aplicada. Belo Horizonte. Ed. UFMG.

Netemeyer, R. G. Bearden, W. O.; Sharma, S. Scaling procedures. London; Sage. 2003.

Orssatto, C. H. (2012) A formulação das estratégias da empresa em um ambiente de aglomeração industrial. Tese (doutorado) apresentada à Universidade Federal de Santa Catarina, Centro Tecnológico. Programa de Pós-Graduação em Engenharia de Produção.

Paim, N. A.; Lara, J. E.; Christino, J.M.M. (2011) Relações quantitativas entre orientação para o mercado, desempenho organizacional $e$ a percepção externa de sucesso em instituições de pesquisas tecnológicas afiliadas à ABIPTI. Revista de Administração Contemporânea - versão online. vol.15 no.6 Curitiba Nov./Dez.

Porter, M (1993). A vantagem competitiva das nações. Rio de Janeiro: Editora Campus.

Porter, M (2002). Competição: estratégias competitivas essenciais. 2. ed. Rio de Janeiro: Campus.

Roselino, J. E. (2006). A indústria de software: o "modelo brasileiro" em perspectiva comparada. Tese de Doutorado do Instituto de Economia, Universidade Estadual de Campinas, Campinas, SP, Brasil.

Santos, G. A. G. dos; Diniz, E. J.; Barbosa, E. K (2004, dezembro). Aglomerações, arranjos produtivos locais e vantagens competitivas locacionais. Revista do BNDES. Rio de Janeiro, v. 11, n. 22, p. 151-179.

Sebrae (2010). 1a. Pesquisa do Perfil dos Profissionais de Tecnologia da Informação. Disponível em www.sebraemg.com.br Acesso em 01/11/2010.

Silveira, A.; Garces, J. (2002, outubro - dezembro). Gestão pública orientada para resultados no Brasil. Revista do Serviço Público. Ano 53 Número 4.

Siqueira, C. L. (2009) Gestão pública orientada para resultados: avaliando o caso de Minas Gerais. Dissertação de Mestrado apresentada à EBAPE/FGV p. 5.

Tabachnick, B. G.; Fidel, L. S. (1966) Using Multivariate Statistics. Harper Collins: New York. 1966

Villaschi Filho, A.; Campos, R (2002). Sistemas/arranjos produtivos localizados: conceitos históricos para novas abordagens. in: Castilhos, C.C. (Coordenadora) Programa de apoio aos sistemas locais de produção: a construção de uma política pública no RS. Porto Alegre: FEE / SEDAI, p. 11-48. 